\title{
Assessment of chest pain in hypertrophic cardiomyopathy using exercise thallium-20I myocardial scintigraphy
}

\author{
DAVID PITCHER, RAY WAINWRIGHT, MICHAEL MAISEY, \\ PAUL CURRY, EDGAR SOWTON
}

From the Departments of Nuclear Medicine and Cardiology, Guy's Hospital, London

SUMMARY Exercise thallium-201 myocardial scintigraphy was performed in 23 patients with hypertrophic cardiomyopathy. Eighteen patients presented with chest pain which was a persistent symptom in 11. Selective coronary arteriography was performed in 16 patients and showed normal coronary arteries in 15 and insignificant luminal irregularities in one patient.

Eighteen patients had abnormal scintigrams. Three had an abnormal distribution of tracer entirely attributable to asymmetric septal hypertrophy, whereas 15 had discrete tracer uptake defects which could not be explained solely by myocardial hypertrophy. In this latter group of patients three scintigraphic patterns were identified: (1) in 10 patients defects were seen in scintigrams immediately after exercise but not in delayed images obtained four to six hours later. Eight of these patients had chest pain. (2) Four patients had uptake defects seen in both initial and delayed images. One patient had chest pain. (3) In three patients, one of whom had chest pain, tracer defects were seen only in delayed images and were not apparent in the initial scintigrams.

Chest pain occurred in eight out of 10 patients with scintigraphic evidence of myocardial ischaemia but was present in only three out of 13 patients with non-ischaemic scintigrams.

The value of exercise thallium-201 myocardial imaging as a diagnostic technique in hypertrophic cardiomyopathy appears limited. Scintigraphic evidence of regional myocardial ischaemia in the absence of significant coronary artery disease, however, contributes to an understanding of the mechanism of angina production in patients with hypertrophic cardiomyopathy.

Despite angiographically normal or dilated coronary arteries, anginal chest pain is a dominant symptom in many patients with hypertrophic cardiomyopathy. ${ }^{1}$ Exercise thallium-201 ( $\left.{ }^{201} \mathrm{Tl}\right)$ myocardial scintigraphy has been shown to be a sensitive technique for detecting abnormalities of regional myocardial perfusion in patients with angina pectoris caused by coronary artery disease. ${ }^{2} 3$ This technique has also been used for the detection of asymmetric septal hypertrophy in patients with hypertrophic cardiomyopathy. ${ }^{4}$ Chest pain in hypertrophic cardiomyopathy, however, has not been studied previously using myocardial scintigraphy. In this communication we report the findings on thallium-201 myocardial scintigraphy in patients with hypertrophic cardiomypathy, and their relation to chest pain.

Received for publication 6 May 1980

\section{Patients and methods}

Twenty-three patients (17 male, six female, aged 26 to 68 years) with hypertrophic cardiomyopathy were studied. Eighteen patients presented with angina pectoris, which was a persisting symptom at the time of investigation in 11. All patients had a full clinical examination, resting 12-lead electrocardiogram, and chest $x$-ray. No patient had hypertension or aortic valve disease. The diagnosis of hypertrophic cardiomyopathy was established by echocardiography and contrast left ventriculography.

Echocardiography showed asymmetric septal hypertrophy (septal to posterior wall ratio greater than 1.3) in 22 patients. Echocardiographic evidence of left ventricular outflow obstruction (mitral systolic anterior motion and/or premature aortic 
valve closure) was present in 11 . One patient had echocardiographic evidence of concentric left ventricular hypertrophy in the presence of a family history of hypertrophic cardiomyopathy and a diagnostic angiographic appearance.

At cardiac catheterisation a left ventricular intracavity pressure gradient was detected in 10 patients at rest and in a further three after isoprenaline stimulation. Ten patients had no resting gradient and did not receive isoprenaline.

Contrast left ventricular angiography showed systolic obliteration of the ventricular cavity in 14 patients. Varying degrees of septal and papillary muscle hypertrophy were seen in all 23 patients. Mild mitral regurgitation was present in seven patients and a moderate degree in one. In 15 patients normally patent coronary arteries were demonstrated by selective arteriography. One patient had minor coronary luminal irregularities which were not considered significant. Seven patients without persistent chest pain did not have selective coronary arteriography.

\section{MYOCARDIAL IMAGING}

All patients underwent a graded maximal exercise test on a bicycle ergometer with continuous monitoring of the electrocardiogram using a single electrode in the $\mathrm{V} 5$ position. $1.5 \mathrm{mCi}{ }^{201} \mathrm{Tl}$ was given intravenously at the limiting end-point of exercise (which was angina pectoris in five patients) and submaximal exercise was continued for two minutes.

Myocardial imaging was performed with a standard field of view gamma camera (Ohio Nuclear Series 100 or 120) equipped with a high sensitivity, parallel-hole collimator. Immediately after exercise myocardial images were obtained routinely in all patients in four standard projections (anterior, left anterior oblique $45^{\circ}$ and $55^{\circ}$, and left lateral) and further myocardial images were acquired in selected views four to six hours after the initial administration of tracer. 200000 counts were recorded in each projection. The images were digitised and stored for later retrieval using a dedicated minicomputer (Digital Equipment Corporation, Gamma 11). A 50 per cent background erase and interpolation was applied routinely to all images.

Images were displayed on a television monitor in 16 grades of colour, linearly related to count density. Analysis was performed by two independent observers. The images were classified as normal if the distribution of tracer was homogeneous and if the outline of left ventricular myocardial tracer activity appeared of normal shape and symmetry. In addition to any observed abnormalities of shape or symmetry, regions of relatively

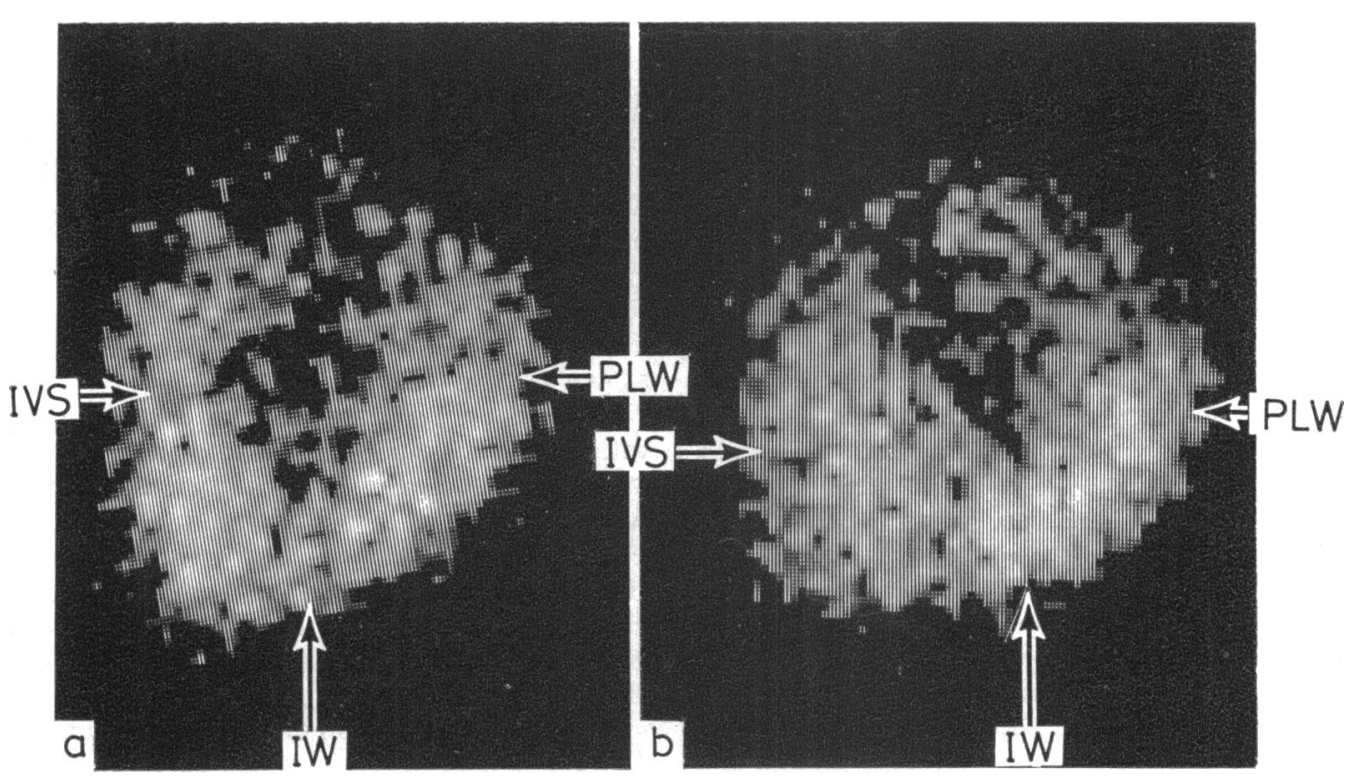

Fig. 1 LAO $45^{\circ}$ scintigrams from (a) a normal subject and (b) a patient with hypertrophic cardiomyopathy. The increased width of septal tracer activity and abnormal image shape in (b) are attributable to asymmetric septal hypertrophy. IVS, interventricular septum; IW, inferior wall; PLW, posterolateral wall. 


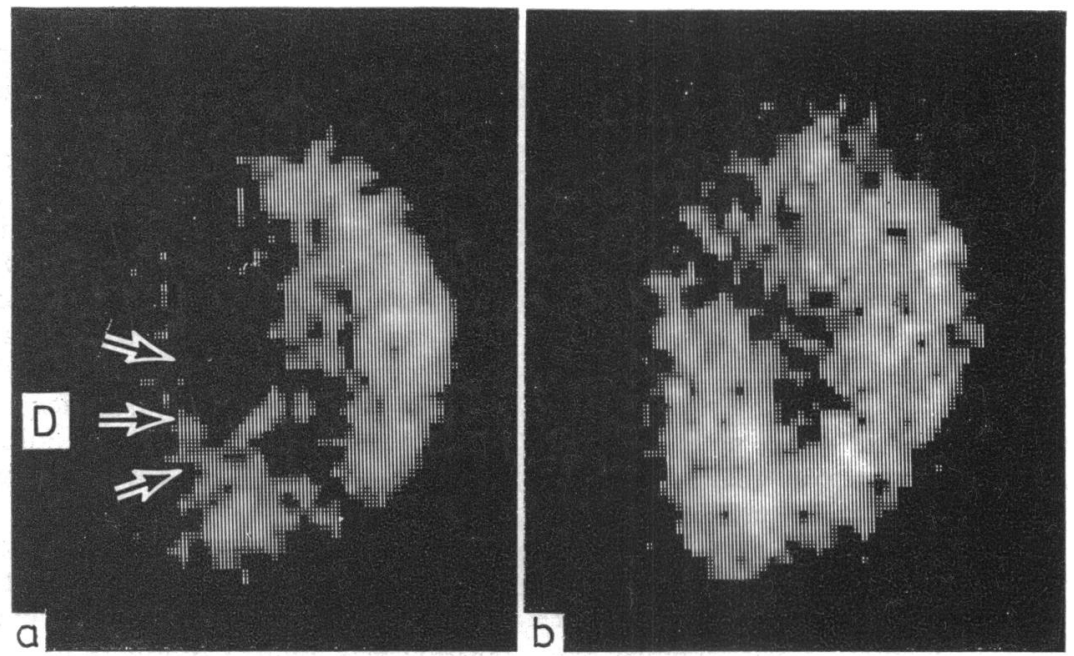

Fig. $2 L A O 45^{\circ}$ scintigrams from a patient with hypertrophic cardiomyopathy and normal coronary arteries (a) immediately after tracer administration during peak exertion and (b) four hours later. $A$ defect of tracer uptake (D) is seen in the septum in (a). Redistribution of thallium to this region seen in the delayed image suggests that the defect was the result of regional myocardial ischaemia.

diminished tracer activity were identified and classified according to their site (septal, apical, inferior, anterolateral, or posterolateral).

The prevalence of each of four symptoms (angina, dyspnoea, palpitation, and syncope) was compared with the observed scintigraphic patterns. Fisher's exact test was used to assess the statistical significance of the results.

\section{Results}

\section{SCINTIGRAPHIC APPEARANCES}

A normal intramyocardial distribution of tracer was seen in five patients, one of whom had chest pain. Abnormal myocardial scintigrams were found in 18 patients. Three patients did not have uptake defects though the septum was clearly thicker than the posterolateral wall in the LAO projection (Fig. 1). Discrete tracer uptake defects not attributable to abnormal anatomy alone were found in the remaining patients and could be classified into three types: (1) the initial images of 10 patients (group 1) showed discrete areas of diminished tracer accumulation which later became normal in delayed scintigrams (Fig. 2): eight of these patients had chest pain; (2) in four patients (group 2) tracer defects were present in the initial scintigrams and persisted in delayed images and were therefore regarded as fixed defects (Fig. 3): one of these patients had chest pain; (3) finally in three patients, one of whom had chest pain, septal tracer defects appeared in the delayed images which had not been present in the initial scintigrams (Fig. 4). The distribution of the observed defects is shown in the Table.

\section{PREVALENCE OF SYMPTOMS}

The prevalence of anginal chest pain was significantly greater $(p<0.01)$ in patients in group 1 than in the remainder of the patients studied (Fig. 5). The prevalence of other symptoms (dyspnoea, palpitation, and syncope) was not significantly greater in any of the groups of patients described.

\section{COMPARISON WITH}

\section{ELECTROCARDIOGRAMS}

There was no significant difference in the prevalence of voltage criteria for left ventricular hypertrophy between any of the groups of patients. Abnormal $Q$ waves were present in 10 patients. Five of these had septal tracer defects (groups 1 or 2) and inferior or inferolateral $Q$ waves. Of the other five, three had no tracer defects, one had an apical defect, and one a septal tracer defect in the delayed images only (group 3 ).

When the distribution of resting ST segment and $T$ wave abnormalities was compared with that of tracer defects 15 out of 19 defects in groups 1 and 2 had corresponding ST/T abnormalities. The three patients in group 3 (reduced septal tracer 

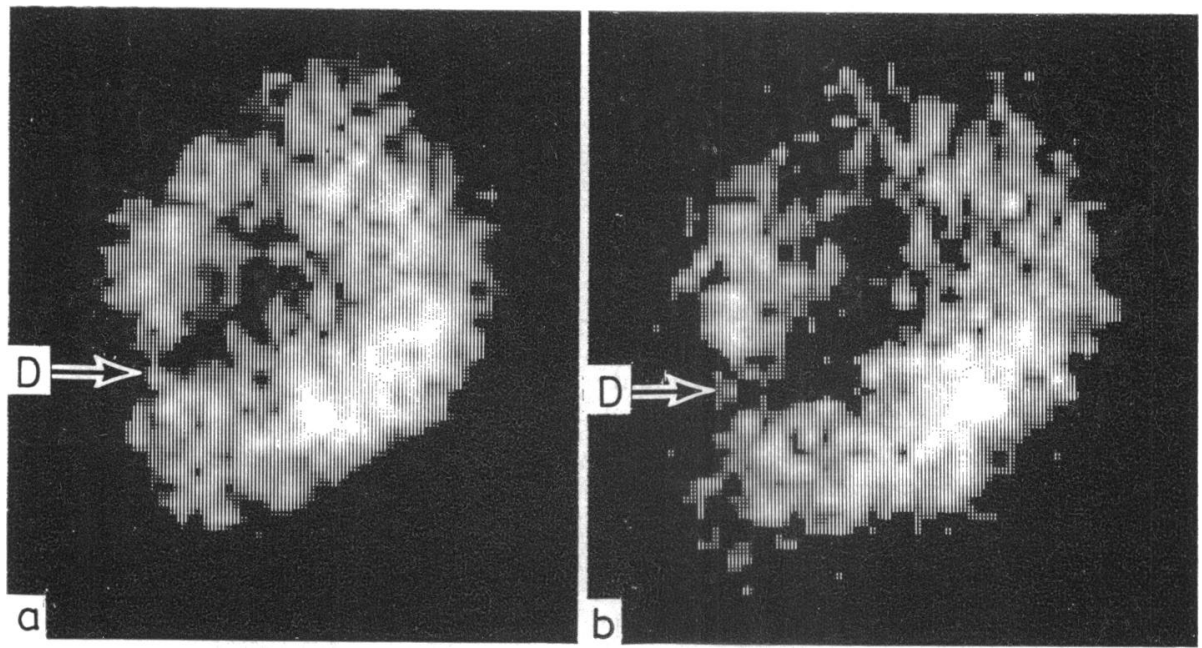

Fig. $3 L A O 45^{\circ}$ scintigrams from a patient with hypertrophic cardiomyopathy (a) immediately after tracer administration and (b) four hours later. The defect in tracer uptake (D) in the lower septum in $(a)$ is seen as a persistent abnormality in $(b)$.

activity on delayed images only) had no corresponding septal ST-T changes but showed such changes in the inferior and lateral leads. Resting ST-T abnormalities occurred in regions without tracer defects in 17 patients including eight with no defects on their scintigrams.

Only two patients showed at least $2 \mathrm{~mm} \mathrm{ST}$ segment depression in lead V5 during exercise. One had the severe septal defect shown in Fig. 2 and the other a fixed septal tracer defect.

\section{COMPARISON WITH ECHOCARDIOGRAMS} AND CATHETER FINDINGS

Septal thickness and septal/posterior wall ratio
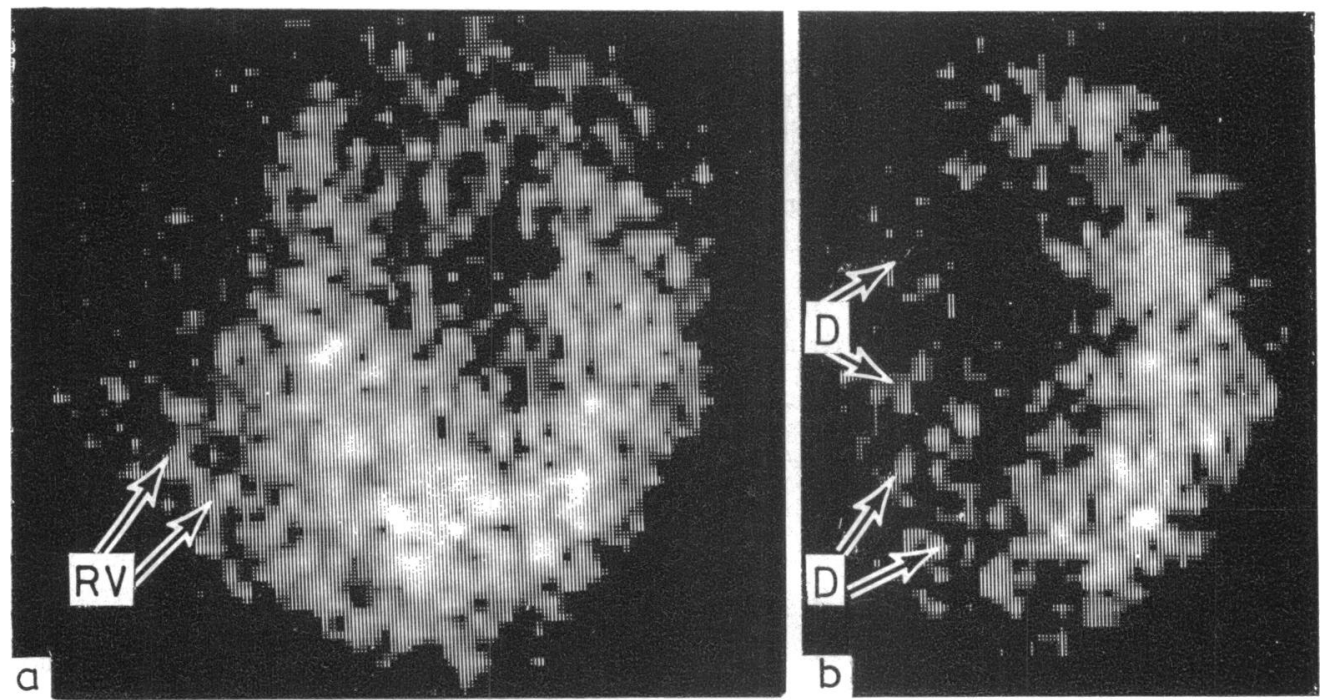

Fig. $4 L A O 45^{\circ}$ scintigrams in another patient with hypertrophic cardiomyopathy (a) immediately and (b) four hours after tracer administration. No tracer uptake defect is visible in (a), but diminished tracer activity (D) in the septum is seen in the delayed image. There is also increased uptake of tracer by the right ventricular myocardium $(R V)$ in this patient. 
measured from the echocardiogram were compared in the groups of patients with the different scintigraphic patterns described. There was no significant difference in these measurements among the various groups, and in particular no significant difference was seen when group 1 was compared with the remainder.

Echocardiographic evidence of left ventricular outflow obstruction occurred in six patients from group 1 and in five other patients. Similarly there was no significant difference between group 1 and the remainder when left ventricular intracavity gradients and left ventricular end-diastolic pressure recorded at cardiac catheterisation were examined.

Estimation of septal and posterolateral wall thickness from the left anterior oblique $45^{\circ}$ scintigrams was prevented by tracer deficits in the region in half of the patients. In the remainder the lack of edge definition prevented accurate or consistent measurements.

\section{Discussion}

Myocardial imaging using ${ }^{201} \mathrm{Tl}$ may be used to detect regional myocardial ischaemia caused by coronary artery disease. ${ }^{2} 3$ Uptake of this tracer by myocardial cells in any region is dependent largely on coronary blood flow at the time of tracer administration. ${ }^{5}$ During exercise coronary flow increases, unless restricted, for example, by a proximal coronary artery stenosis. Thus, when ${ }^{201} \mathrm{Tl}$ is given during exercise its delivery to myocardium beyond such a stenosis is restricted, while delivery to unaffected regions is increased. Furthermore, increased oxygen demand during exercise may lead to myocardial hypoxia distal to the stenosis. Since ${ }^{201} \mathrm{Tl}$ uptake into myocardial cells is by active, ATP-dependent transport requiring oxygen, tracer

Table Types and distribution of tracer defects seen in 15 patients

\begin{tabular}{llllll}
\hline & & \multicolumn{4}{l}{ No. of distinct defects } \\
\cline { 5 - 6 } $\begin{array}{l}\text { Observed } \\
\text { abnormality }\end{array}$ & patients & Septal & Apical & Inferior & Total \\
\hline $\begin{array}{c}\text { Ischaemic defects } \\
\text { seen on initial } \\
\text { but not on } \\
\text { delayed images }\end{array}$ & 10 & 8 & 4 & 2 & 14 \\
$\begin{array}{c}\text { Fixed defects } \\
\text { seen on both } \\
\text { initial and } \\
\text { delayed images }\end{array}$ & 4 & 3 & 1 & 0 & 4 \\
$\begin{array}{c}\text { Late defects } \\
\text { seen only on } \\
\text { delayed images }\end{array}$ & 3 & 3 & 0 & 0 & 3 \\
\hline
\end{tabular}

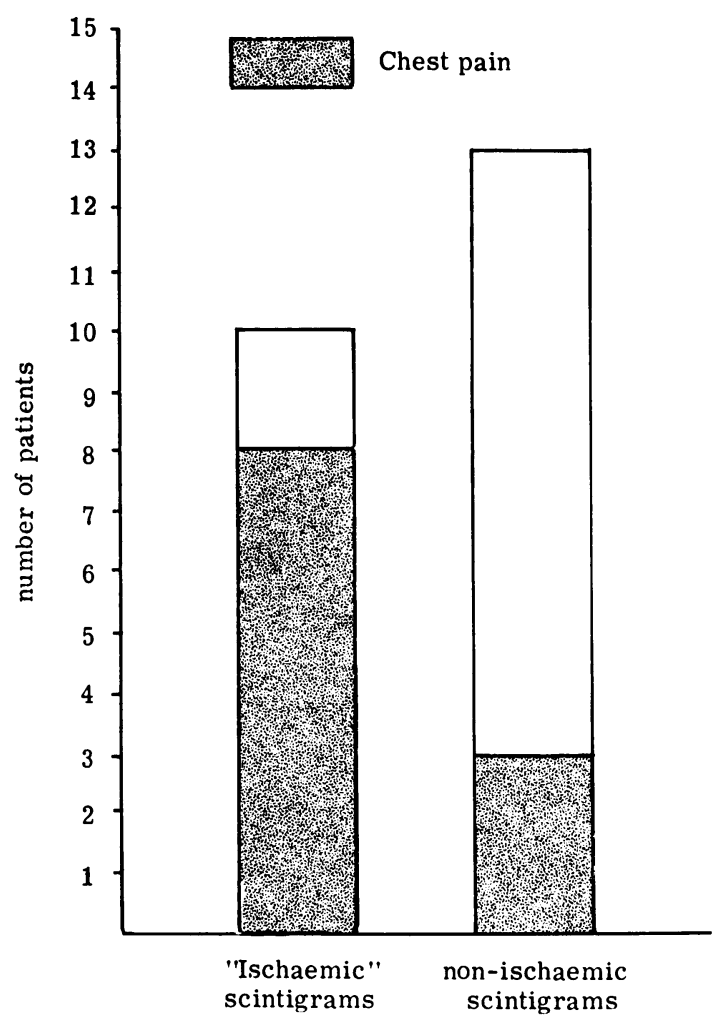

Fig. 5 Prevalence of angina in patients with scintigraphic evidence of regional myocardial ischaemia (group 1) and in those with non-ischaemic scintigraphic appearances. The differences are statistically significant $(p<0.01)$.

uptake is impaired by hypoxia, which further enhances the relative paucity of tracer accumulation in the ischaemic zone. This is seen as an area of relatively diminished tracer activity on myocardial images acquired immediately after ${ }^{201} \mathrm{Tl}$ administration during exercise. Over the next few hours the isotope is redistributed, both to previously ischaemic myocardium and to extracardiac organs. Repeat imaging several hours after tracer administration allows differentiation of transiently ischaemic myocardium from a region of previous infarction ${ }^{6}$ which will not show accumulation of tracer activity in delayed images.

In hypertrophic cardiomyopathy additional factors are encountered which may affect scintigraphic appearances. Septal thickness is increased and may be seen as a wider band of tracer activity when seen in profile. Septal motion, however, is reduced and posterior wall motion often increased. 
Since ${ }^{201} \mathrm{Tl}$ scintigrams integrate both systolic and diastolic events the profiled image of the septum will appear relatively thinner, and that of the posterolateral wall relatively wider as a result of these differences in wall motion. Tracer activity in hypertrophied papillary muscles and systolic cavity obliteration may increase apparent wall thickness near the apex. These factors may contribute to an increased apparent septal thickness near the apex, reported by Bulkley et al. ${ }^{4}$ in hypertrophic cardiomyopathy. We consider myocardial scintigraphy to be an inappropriate technique for the measurement of wall thickness, both because of its low resolution and because of the tracer defects seen in many of our patients.

The precise significance of some of these defects is uncertain. Defects seen on the initial images but not on delayed imaging provide evidence, however, of regional myocardial ischaemia during exercise in the absence of coronary artery disease. Anginal chest pain was significantly more common in patients with this type of defect. The distribution of these defects (Table) corresponds with the known distribution of myocardial fibre disarray and hypertrophy in hypertrophic cardiomyopathy, ? but regional ischaemia cannot be attributed to myocardial hypertrophy alone. We found ischaemic scintigraphic defects in some of our patients, but not in others with an equal degree of hypertrophy. We suggest that regional myocardial ischaemia may occur when localised abnormalities of contraction and relaxation generate high intramyocardial pressures in both systole and diastole, and thereby reduce local coronary flow. Since the greater part of coronary flow occurs in diastole, abnormalities of left ventricular relaxation are probably of greatest importance in the genesis of myocardial ischaemia. ${ }^{8}$ This is emphasised by the increased incidence of angina pectoris associated with impaired left ventricular filling in hypertrophic cardiomyopathy. ${ }^{9}$

Two other types of tracer defect were observed in our patients. Fixed defects, present in both initial and delayed images, probably represent areas of myocardial scarring, known to occur in hypertrophic cardiomyopathy. ${ }^{10}$ Alternatively these defects might be the result of severe abnormalities of regional contraction and relaxation, sufficient to maintain ischaemia at rest and prevent redistribution of ${ }^{201} \mathrm{Tl}$ to a region with diminished uptake on the initial scintigrams. Septal tracer defects, however, seen in three patients in the delayed images only, cannot be attributed to septal ischaemia since there was no impairment of local ${ }^{201} \mathrm{Tl}$ uptake in the initial images. The subsequent appearance of relatively reduced tracer activity in the septum after several hours may be the result of more rapid tracer elimination from the septum than from the posterolateral wall. Since the regional clearance of ${ }^{201} \mathrm{Tl}$ from the myocardium is independent of blood flow, ${ }^{11}$ a more rapid leakage of isotope from abnormal septal myofibrils would provide a possible explanation for this phenomenon. Alternatively changes in left ventricular geometry or wall motion may give rise to an asymmetry of tracer activity, even if the intramyocardial distribution is homogeneous. The three patients with this scintigraphic pattern had no clinical or other investigatory features to distinguish them from the remainder of the patients in the study.

Thus, our findings indicate that at least three abnormal scintigraphic patterns, not attributable to myocardial hypertrophy alone, may be found in patients with hypertrophic cardiomyopathy. In particular, scintigraphic evidence of regional myocardial ischaemia during exercise was found in several patients and offers an explanation for angina pectoris in this condition. Regional myocardial ischaemia could not be identified from the resting electrocardiograms and single lead exercise electrocardiography was a relatively insensitive indicator of ischaemia in this group of patients. Further studies are indicated to establish the clinical and in particular the prognostic significance of scintigraphic abnormalities in hypertrophic cardiomyopathy.

\section{References}

1 Frank S, Braunwald E. Idiopathic hypertrophic subaortic stenosis. Clinical analysis of 126 patients with emphasis on the natural history. Circulation 1968; 37: 759-88.

2 Bailey IK, Griffith LSC, Rouleau J, Strauss HW, Pitt B. Thallium-201 myocardial perfusion imaging at rest and during exercise. Comparative sensitivity to electrocardiography in coronary artery disease. Circulation 1977; 55: 79-87.

3 Wainwright RJ, Maisey MN, Sowton E. Segmental quantitative thallium scintigraphy in diagnosis of coronary artery disease-correlation with coronary arteriography and exercise stress testing (abstract). Br Heart $\mathcal{F}$ 1978; 40 : 447.

4 Bulkley BH, Rouleau J, Strauss HW, Pitt B. Idiopathic hypertrophic subaortic stenosis; detection by thallium-201 myocardial perfusion imaging. $N$ Engl $f$ Med 1975; 293: 1113-6.

5 Strauss HW, Harrison K, Langan JK, Lebowitz E, Pitt B. Thallium-201 for myocardial imaging: relation of thallium-201 to regional myocardial perfusion. Circulation 1975; 51 : 641-5.

6 Pohost GM, Zir LM, Moore RH, McKusick KA, Guiney TE, Beller GA. Differentiation of transiently ischemic from infarcted myocardium by serial imaging after a single dose of thallium-201. Circulation 1977; 55: 294-302. 
7 Roberts WC, Ferrans VJ. Pathologic anatomy of the cardiomyopathies. Hum Pathol 1975; 6: 287-342.

8 Sanderson JE, Gibson DG, Brown DJ, Goodwin JF. Left ventricular filling in hypertrophic cardiomyopathy. An angiographic study. Br Heart $\mathcal{F} 1977$; 39: 661-70.

9 St. John Sutton MG, Tajik AJ, Gibson DG, Brown DJ, Seward JB, Giuliani ER. Echocardiographic assessment of left ventricular filling and septal and posterior wall dynamics in idiopathic hypertrophic subaortic stenosis. Circulation 1978; 57: 512-20.

10 Maron BJ, Epstein SE, Roberts WC. Hypertrophic cardiomyopathy and transmural myocardial infarction without significant atherosclerosis of the extramural coronary arteries. Am $\mathcal{F}$ Cardiol 1979; 43: 1086-102.

11 Gerwitz H, O'Keefe DD, Pohost GM, Strauss HW, McIlduff JB, Daggett WM. The effect of ischemia on thallium-201 clearance from the myocardium. Circulation 1978; 58: 215-9.

Requests for reprints to Dr David Pitcher, Plymouth General Hospital, Plymouth, Devon. 